\title{
Circulación de conocimientos y prácticas médicas en el Reino Latino de Jerusalén: algunas reflexiones sobre su estudio
}

\section{Esteban Greif}

Consejo Nacional de Investigaciones Científicas y Técnicas (CONICET) / Facultad de Filosofía y Letras, Universidad de Buenos Aires, Argentina

estebangreif1184@gmail.com

Recibido: 18-02-2020. Aceptado: 08-06-2020.

\begin{abstract}
Resumen
El mundo creado por los francos en Tierra Santa luego de la conquista europea de Jerusalén ha sido un escenario sobre el que desde comienzos del siglo XX los historiadores se han detenido a la hora de dilucidar los circuitos y las formas de circulación de conocimiento entre el mundo del Mediterráneo oriental y el continente europeo. El estudio de la medicina desarrollada en la sociedad latina del Reino de Jerusalén forma parte del conjunto de los trabajos que atendieron a este capítulo de la historia del conocimiento del mundo Mediterráneo medieval. El recorrido y la reflexión sobre su evolución historiográfica nos permitirán señalar a grandes rasgos las principales líneas de análisis y las formas de abordar este campo específico, así como los grandes cambios y las interpretaciones novedosas para comprender este aspecto de la historia de las cruzadas. Del mismo modo, será posible señalar algunas de las dificultades en algunos de los trabajos que abordaremos, así como posibles perspectivas para aproximaciones futuras.
\end{abstract}

Palabras clave: historiografía, medicina, cruzadas, lepra.

Circulation of Knowledge and Medical Practices in the Latin Kingdom of Jerusalem: Some Reflections on its Study

\begin{abstract}
The society founded by the Franks in the Holy Land after the European conquest of Jerusalem has been the subject of study for many historians who, since the beginnings of the 2oth century, tried to elucidate the ways knowledge circulated between the Mediterranean East and the European continent. The study of the medical practice developed in the Latin society of the Kingdom of Jerusalem belongs to the set of investigations on this particular chapter of the history of knowledge in the Medieval Mediterranean World. The study and reflection on its historiographical evolution will
\end{abstract}


allow us to outline, in general terms, the main lines of analysis and ways of approaching this specific field, as well as the great changes and novel perspectives on the issue in order to understand this aspect of the history of the crusades. Likewise, it will be possible to identify possible difficulties in the works addressed, as well as to point out perspectives for future proposals.

Keywords: historiography, medicine, crusades, leprosy.

\section{Introducción: medicina en las cruzadas}

Los estados cruzados resultan un espacio propicio para el estudio de la circulación de conocimientos y prácticas provenientes de tradiciones sumamente diversas. Este aspecto no fue inadvertido por los historiadores de la medicina que desde comienzos del siglo XX se dedicaron al estudio de la práctica médica y de las instituciones dedicadas al cuidado de los enfermos en el Reino Latino de Jerusalén (Walsh, 1919). Conformada por algunas monografías generales (Wickersheimer, 1951; Woodings, 1971), dicha temática tuvo un crecimiento lento. ${ }^{1}$ En efecto, los estudios que abordaron sistemáticamente este campo particular comenzaron a surgir hacia mediados del siglo pasado y cobraron un ímpetu mayor en las últimas tres décadas (Edgington, 1994; Kedar, 1998; Ell, 1996; Ficarra, 1996; Dolev, 1996). De esta manera pudo consolidarse una tradición de estudios que abordó diversos aspectos de la historia de la medicina en las cruzadas.

Dicha tradición se refleja en la publicación de dos libros, únicos tratamientos completos del tema, en años recientes. El primero de ellos, Medicine in the Crusades, fue publicado en el año 2004 por Piers Mitchell, mientras que el segundo, Die Seuchen der Kreuzzüge, por Thomas Gregor Wagner, en el año 2009. El objetivo del primero consistió en analizar el estado de la práctica médica en los estados cruzados que desarrollaron los sujetos e instituciones creadas por los francos en Outremer. En este sentido, el libro se ocupa de los médicos que ejercieron en Tierra Santa, así como los hospitales sostenidos por las órdenes militares. Su trabajo, además, presta especial atención al tratamiento de las heridas y las prácticas quirúrgicas que tuvieron lugar entre los profesionales locales y europeos. El libro de Wagner, por otro lado, se enfoca mayormente en el entendimiento de la enfermedad y las condiciones epidemiológicas que afectaron a los ejércitos cruzados.

Sumado a estas dos obras de conjunto, recientemente aparecieron otros trabajos que de manera similar abordaron diversas temáticas de la teoría y la práctica médica en las cruzadas, como, por ejemplo, el artículo de Susan Edgington titulado Oriental and occidental medicine in the crusader states. Publicado en el año 2011, destacaba por su presentación sistemática y actualizada de los contenidos generales sobre la historia de la medicina en las cruzadas (Edgington, 2011).

Al margen de estos abordajes generales, toda una serie de trabajos se ocupó de diversos aspectos de la historia de la medicina en las cruzadas. Señalaremos los aspectos sobresalientes de dichos estudios y su análisis de la circulación de conocimientos y prácticas médicas en el Reino Latino de Jerusalén bajo una división temática que responde a los grandes tópicos en los que este campo se divide. A saber, 1) el estudio de los hospitales creados por las órdenes militares,

1 El trabajo monográfico de Ernest Wickesheimer en 1951 fue el primer tratamiento completo de la temática. En este estudio, fueron señalados los ejes principales por los que circularían las investigaciones posteriores. De la misma forma, el artículo de Anne Woodings (1971) también fue articulador de las producciones siguientes, convirtiéndose en un trabajo fundamental para el estudio de la medicina en las cruzadas. 
fundamentalmente el de los caballeros hospitalarios; 2) el rol de los médicos y el grado de desarrollo teórico de la medicina existente en las sociedades de Ultramar; 3) los trabajos sobre la legislación médica en Outremer y 4) el impacto social del padecimiento de algunas enfermedades y su tratamiento, en especial el de la lepra.

\section{Las órdenes militares y la práctica médica}

En relación a las instituciones que desplegaron algún tipo de atención médica, los trabajos del área se detuvieron fundamentalmente en el papel de los caballeros de San Juan de Jerusalén y los hospitales que la orden sostuvo (Barber, 2000; Edgington, 1999; Hume, 1940; Kedar, 1998; Miller, 1978). En cambio, menos se ha indagado acerca la actividad médica de las restantes órdenes militares que surgieron durante las cruzadas en Ultramar (Mitchell, 2007). Sin embargo, en torno al tema que nos ocupa, un aspecto atendido en más de uno de estos trabajos fue el origen de los conocimientos y prácticas médicas que orientaron la atención de los hospitales sostenidos por las órdenes.

Según el conocimiento médico medieval, la prescripción de determinados alimentos servía para la restauración del equilibrio humoral del enfermo y, así, la recuperación de su estado de salud. Por tal motivo, no es llamativa la centralidad otorgada por los hermanos de las órdenes militares a la indicación de diferentes comidas como un procedimiento terapéutico para la curación de los pacientes. ${ }^{2}$ Dicha indicación ha sido al mismo tiempo la herramienta principal para señalar la procedencia de los conocimientos médicos adoptados por las órdenes. De esta forma, las coincidencias entre las comidas prescritas en diversos tratados que circulaban en el mundo medieval con las que figuraban en los estatutos de las órdenes militares le sirvieron a más de un historiador para indicar posibles modelos de inspiración médica y organización terapéutica.

De tal manera, Indrikis Sterns pudo señalar que las terapias dispensadas a los enfermos en los hospitales de las órdenes militares en Ultramar se inspiraban en las prescripciones alimentarias del Regimen sanitatis salernitanum. El autor registró numerosas similitudes terapéuticas en torno a la administración de alimentos entre este tratado y los estatutos de las órdenes militares en Tierra Santa, factor que evidenciaba para él la centralidad de la escuela italiana en la organización médica de los hospitales de dichas órdenes (Sterns, 1983; Mitchell, 2004, pp. 99-103; Edgington, 1998).

El mismo procedimiento fue adoptado años después por Susan Edgington y Piers Mitchell quienes señalaron mayor cantidad de puntos de coincidencia entre las dietas prescritas en los estatutos de las órdenes y las recomendadas en textos de origen oriental. Mientras que para Edgington la procedencia de las terapias adoptadas por los hospitalarios se basaron en los textos árabes que circulaban en el Mediterráneo, como el tratado del médico persa al-Majusi (ì?- ca. 994) titulado kitab al-malaki (Libro del arte médico) (Edgington, 1998), para Mitchell el modelo terapéutico derivaba del libro Dieta para el Emperador del médico bizantino Oribasio de Pérgamo (Mitchell, 2004, pp. 99-103). En ambos casos, sus trabajos revisaban lo propuesto por Timothy Miller en su conocido artículo The Knights Hospitallers and the Latin West (1978). La propuesta de este último insistía también en la adopción de un modelo bizantino de organización hospitalaria, aunque no se basaba en la comparación de prescripciones dietarias, sino en los servicios otorgados en las instituciones griegas y sus similitudes 
con el hospital de San Juan de los caballeros hospitalarios. Su propuesta, más allá de las críticas que recibió (Edgington, 1998; Luttrell, 1994), tenía la virtud de orientar la comparación sobre los aspectos institucionales en lugar de los teóricos.

Pese a las importantes contribuciones que brindaron los análisis de Mitchell, Edginton o Sterns sobre nuestro conocimiento de la práctica médica desplegada por las órdenes militares en Tierra Santa, su procedimiento para indicar la procedencia de las terapias desplegadas por los hermanos de dichas órdenes presentaría una serie de dificultades.

En primer lugar, y en torno a la información que brindan los estatutos y otros documentos de la orden de los hospitalarios, la dieta responde a consignas que caen fuera de lo "estrictamente médico". Las horas litúrgicas de cada jornada, así como las festividades y otros días del calendario religioso, influían enormemente en los alimentos y las bebidas que podían ser o no suministradas al conjunto de los residentes en el complejo de la orden (Edgington, 2005a).

En segundo lugar, si a esto sumamos el hecho de que el acervo teórico-práctico tanto de los médicos occidentales como de los orientales era esencialmente el mismo -la medicina galeno-hipocrática-, pierde fuerza el sentido de una comparación de modelos procedentes de diferentes espacios como estructuradores de diferentes tipos de teoría médica, sea esta de origen persa o bizantino. ${ }^{3}$ De la misma forma, podríamos preguntarnos si los caballeros de San Juan y las restantes órdenes militares se basaron para la redacción de sus estatutos en más de un autor y no solo en aquellos con los que los historiadores encontraron mayores similitudes teóricas entre los alimentos prescriptos en sus textos médicos y los documentos hospitalarios (Siraisi, 1990, pp. 52-53).

Lo expuesto hasta aquí permitiría pensar que, a la hora de comparar documentos, podría resultar conveniente enfocarnos sobre los aspectos institucionales concretos que organizaban la práctica hospitalaria en diferentes instituciones. Por ejemplo, la disposición de profesionales médicos, la estructura edilicia de los hospitales o las terapias que expresamente adoptaron los hermanos de las órdenes que existían en los hospitales del mundo griego en Tierra Santa. En efecto, esta fue la forma en la que procedieron otros historiadores que pudieron identificar numerosos elementos de la cultura hospitalaria bizantina replicada en las instituciones creadas por las órdenes militares (Amouroux, 1999; Touati, 2007).

\section{Acerca de los médicos y de la teoría médica en las Cruzadas}

Diversas fuentes de la historia de las cruzadas y el Reino Latino de Jerusalén describen la importante presencia de médicos europeos dedicados a la atención y cuidado de los enfermos en los estados cruzados, así como la existencia de numerosos profesionales de origen oriental que ejercieron en Tierra Santa durante la ocupación latina (Wickersheimer, 1936; Mitchell, 2004, pp. 17-40). Los debates en torno al grado de formación teórica y práctica que poseían unos y otros han dado lugar a numerosos trabajos comparativos acerca del desarrollo alcanzado en Occidente y en Oriente en materia médica al comienzo de las cruzadas. Del mismo modo, se ha discutido el carácter de los intercambios culturales entre estos dos espacios. 
Uno de los primeros autores en detenerse sobre el tema fue Ernst Wickersheimer (1951, 1936). En sus clásicos trabajos, el autor daba cuenta de los médicos europeos de mayor prestigio que actuaron y vivieron en el Reino de Jerusalén. Más de 50 años después de la aparición del trabajo de Wickersheimer, Piers Mitchell puso el foco sobre el grupo más amplio y mayoritario de médicos por fuera de aquellos que poseían el título de magister (Mitchell, 2004, p. 11). ${ }^{4}$

Sobre los médicos provenientes del mundo europeo, se ha sostenido que poseían una formación inferior a los de origen oriental. Por esta razón, se ha afirmado también que estos últimos eran preferidos por los nobles y otros miembros de la sociedad latina en Tierra Santa sobre los profesionales europeos. ${ }^{5}$ En este sentido, Claude Cahen podía manifestar que "la preferencia de los cruzados por los médicos indígenas es conocida" (Cahen, 1934, p. 353) mientras que Ernst Wickersheimer señalaba que muchos de estos médicos francos "eran de saber mediocre" y que la nobleza "no tardaría en preferir a sus colegas indígenas" (Wickersheimer, 1951, p. 692). De la misma manera, Francesco Gabrieli en su importante Arab Historians of the Crusades asumía que la medicina practicada por los francos era inferior (Gabrieli, 1969, pp. 76-77), así como lo hacía Ann Woodings, quien afirmaba que la práctica médica en Medio Oriente era ampliamente más avanzada que la europea (Woodings, 1971, p. 268).

Semejantes consideraciones también pueden ser encontradas en estudios más recientes (Elisseef, 1986; D'Alverny, 1982). Afirmaciones de este tipo se basaban en diferentes explicaciones que hacían foco en razones como el contexto de guerra santa de las cruzadas, la ausencia de un gran centro intelectual local en los estados del Oriente latino o la destrucción de las bibliotecas durante las conquistas europeas (Van den Abeele, Tihon y Draelants, 2000).

Sin embargo, los estudios de los últimos años han permitido el reconocimiento de diferentes espacios y personajes del mundo latino de Outremer como protagonistas de un intercambio y producción científica mucho más rica de lo que se había sostenido. De tal forma, desde la década de los 'go del siglo pasado, diferentes trabajos contribuyeron a la conformación de una nueva interpretación en términos científicosculturales de la sociedad creada por los cruzados en el Oriente latino. ${ }^{6}$ Dentro de esta renovación de estudios debemos incluir la circulación y apropiación de saberes en relación con los conocimientos y la formación de los médicos europeos que fueron a las cruzadas. De esta forma, se descarta la idea de rechazo de los médicos francos del aprendizaje de los conocimientos disponibles en la región de Siria y Palestina (Micheau, 2000). El ejemplo de la ciudad de Antioquía, gran centro de producción de textos también en época de las cruzadas, demostraba que la sociedad creada por los europeos en Tierra Santa lejos estaba de desconocer el acervo teórico-cultural local

4 Mientras que palabra medicus podía hacer referencia a los médicos maestros, los términos miege/mire, del francés antiguo, designaban a un conjunto más amplio de profesionales. Si bien los diferentes términos servían para designar diversas jerarquías de especialistas, a veces su uso podía referir a unos y a otros indistintamente. En este sentido, los términos con que eran designados en las fuentes muchas veces dificulta la comprensión del tipo de profesión y las diferencias jerárquicas existentes entre estos. En efecto, comúnmente se hacía uso indistinto de la palabras medicus/miege/mire para referirse a un grupo diverso de profesionales. De hecho, la palabra miege a veces refería a los médicos magister -aquellos que poseían formación universitaria- pero también a boticarios, barberos y sanadores que practicaban distintos tipos de medicina popular (Mitchell, 2004, pp. 11-13).

5 Este tipo de presunciones se sostenían en trabajos que, desde la historia de la ciencia y de la medicina medieval, prestaban atención a la actividad erudita de la España musulmana y de la Italia meridional de los siglos XI y XII, y a la actividad de las primeras universidades europeas. Consideraban que las cruzadas no correspondían a este cuadro general de intercambio cultural entre Oriente y Occidente.Véase, por ejemplo, el artículo de Charles Haskins (1925).

6 Esta renovación historiográfica se expresó, por ejemplo, en las tres reuniones científicas organizadas por la Universidad de Lovaina y las ediciones posteriores de los trabajos presentados en tres libros diferentes que, bajo el título de "East and West in the Crusader states", aparecieron entre 1996 y 2003 (Ciggaar, Teule y Davis, 1996-2003). Asimismo, la serie de trabajos que reúne otro volumen al que ya nos referimos, producto del coloquio también organizado por la Universidad Católica de Lovaina en 1997 (Van den Abeele, Tihon y Draelants, 200o). Véase también Ciggaar (2001). 
ni la producción del mismo (Burnett, 2000; Edgington, 2005c). De la misma forma, trabajos más recientes han puesto en evidencia el interés que poseían los francos por la lectura de textos médicos orientales (Savage-Smith, 2006).

Por otro lado, como señaló Lawrence Conrad, muchos de los análisis que solían destacar la poca relevancia y la escasa formación teórica de los médicos francos se basaban en lecturas sesgadas de ciertos pasajes de diversas fuentes, como las Memorias... de Usama ibn Munqidh (1095-1188), ${ }^{7}$ que dieron lugar a la interpretación de la superioridad y la preferencia por la medicina local ante la practicada por los francos (Conrad, 1999). La idea general que subyace a todas las fuentes de origen árabe es que los europeos eran cultural y socialmente inferiores en numerosos aspectos (Albarrán Iruela, 2017, pp. 193-194; Hillebrand, 1999, pp. 352-354). Semejante juicio de valor sin duda influyó en su conceptualización de los médicos francos (Conrad, 1999, pp. XXXV-XXXVI). En efecto, muchas de las afirmaciones acerca de la superioridad médica oriental partieron de presunciones que no respondían al análisis crítico de las fuentes sino a la creencia compartida en la historiografía de la superioridad de la medicina local (Conrad, 1999, p. XXXI). Por ejemplo, así ocurría en el importante trabajo de Anne Woodings quien sostuvo que la continua contratación de profesionales locales por la nobleza indicaba no solo su superioridad profesional sino también el supuesto rechazo de los médicos francos a las posibilidades de incrementar sus conocimientos y nivelarse así con su contraparte oriental (Woodings, 1971, p. 275)

Sin embargo, también es posible indicar en la literatura de la época pasajes que señalan el sentido contrario a esta presunción. En efecto, si atendemos a los relatos de otros autores, también encontramos descripciones donde los europeos dicen conocer mejor las técnicas y teorías propias del arte de curar. Es decir, relatos en los que los mismos prejuicios o sentimientos de superioridad actúan, aunque en sentido inverso, de la misma forma que lo hacía Usama en sus Memorias (Handerson, 1918, pp. 22-24). En definitiva, asumir la superioridad de uno u otro espacio derivaría de la creencia en uno u otro de los autores. Si a este factor sumamos el hecho de que no existían grandes diferencias entre la atención médica que podían brindar los médicos de un lado y del otro del Mediterráneo, esta presunción pierde todo sentido. Como ya mencionamos las enfermedades, entendidas fundamentalmente como producto de un desbalance de los humores, eran tratadas a partir de modificaciones de la dieta, posteriormente complementada con la aplicación de drogas, baños, sangrías y en última instancia, con el recurso de la cirugía (Mitchell, 2004, p. 57; Mitchell, 2016). Es decir, el acervo básico de ideas y prácticas médicas en el mundo medieval era esencialmente el mismo: la medicina galénica-hipocrática.

\section{Legislación médica en el Reino Latino de Jerusalén}

Los europeos desarrollaron diversos corpus de leyes que legislaban sobre las personas que habitaban los estados cruzados. El libro que regulaba sobre la población burguesa en Tierra Santa se titulaba Livre des Assises de la Cour de Burgeois. Dentro de este corpus de leyes existían tres capítulos sobre legislación médica y veterinaria. ${ }^{8}$ Diversas hipótesis se han sostenido acerca de las fuentes utilizadas en la composición de este texto. Para Joshua Prawer no existió un único modelo de inspiración en la redacción de los Assises de Bourgeois. Sin embargo, aunque no descartó de su análisis la importancia de la tradición local, sostuvo que en su composición el elemento

7 Ver el texto árabe en Hitti (1930) y la traducción al inglés en Hitti (1929).

8 Sobre el autor y la composición del Livre des Assises de Bourgeois véase Prawer (1980, pp. 366-367). 
fundamental era resultado de la costumbre romana traída desde Europa por los nobles del norte de Francia e Italia (Prawer, 1980, p. 360). Gran parte de este texto legislativo se basó, como Prawer señaló, en un código provenzal conocido como Lo Codi, redactado probablemente en Arles c. $1149 .{ }^{9}$ El carácter de "practicidad y plasticidad" del texto provenzal es lo que permitió para el autor su elección y adaptación a la realidad de los estados cruzados (pp. 376-386). ${ }^{10}$

La propuesta de Prawer, a propósito de la sección médica de los Assises, fue recientemente revisada. Como indicó Piers Mitchell, este temprano código romano trataba exclusivamente sobre daños a las propiedades y el valor de las compensaciones que había que pagar en cada caso. De tal modo, las similitudes en relación con la negligencia en el tratamiento médico a esclavos que se señalan en ambos textos no son tan claras. Los Assises son mucho más específicos sobre castigos y tratamientos médicos que los enunciados generales de la LexAquilia. Por lo tanto, para Mitchell la relación que Prawer sugiere no es adecuada. Agregaba además, que “(...) if the Frankish text did indeed have its origins in the LexAquilia, then a considerable degree of evolution had occurred prior to the writing of the Jerusalem code" (Mitchell, 2004, p. 227). De esta manera, se sostuvo que sería más probable que la influencia de la costumbre legal siria hubiera actuado como base para la regulación de las secciones en cuestión (Edgington, 2005b; Mitchell, 2004, p. 227).

Un primer elemento en este sentido deriva de una observación de Piers Mitchell sobre el capítulo de los Assises que se ocupa de regular los deberes y la praxis de los médicos. Dicho capítulo contiene una sección sobre el otorgamiento de licencias a médicos para el ejercicio legal. Semejante práctica, afirma el autor, no existía en Europa antes de las Cruzadas. En cambio, en el mundo islámico se encontraba ampliamente desarrollada. Por tal motivo, Mitchell afirmaba que lo que reflejaba este capítulo era un préstamo que los francos tomaron de la costumbre local. ${ }^{11}$

En el mismo sentido, se ha sugerido sobre esta sección de los Assises que su modelo de inspiración habría sido el manual de legislación civil musulmana llamada hisbah (Edgington, 2005b). Se designaba de esta forma a una serie de textos que contenía un conjunto variado de regulaciones sobre las diferentes actividades que se desarrollaban en las ciudades bajo dominio musulmán. Versaba sobre diferentes asuntos como transacciones comerciales, moral religiosa, seguridad y salubridad en el trabajo, responsabilidad civil de los diferentes profesionales, etc. (Pormann y Savage-Smith, 2007, pp. 85-87).

El oficial a cargo de que se cumpliera lo que dictaban estos manuales era llamado muhtasib, lo que refuerza la idea de que el hisbah era el sustrato de los Assises para la sección médica, ya que en Outremer la responsabilidad de controlar que un médico cumpliera ciertos estándares en su trabajo recaía en un funcionario llamado mathessep. Este último era un oficial que asistía al vizconde de la Cour des Bourgeois y comandaba un cuerpo de hombres armados para cumplir con sus tareas de inspección en las ciudades. ${ }^{12}$ De tal modo, resulta lógica la relación entre el término mathessep, que

9 Lo Codi constituía un tratado de leyes romano cuya influencia fue importante en el sur de Francia y en parte de España. Fue traducido, entre otras lenguas, al latín, al francés, al catalán y al castellano. Tal cantidad de lenguas, según Prawer, demuestra su gran uso, o al menos, el interés constante por los teóricos del derecho en este código provenzal (Prawer, 1980, p. 362).

10 Cfr. Nader (2006, p. 57).

11 Efectivamente, en este capítulo se indicaba que todos los médicos que arribaban a una ciudad franca debían pasar un examen para ejercer su arte. Si aprobaban, se les otorgaba la carta de permiso para ejercer como "médico de orina", es decir, como el médico que poseía el conocimiento necesario para prescribir terapias (Mitchell, 2004, p. 224).

12 Para el mundo latino en Outremer, el primer comentario legal que menciona la existencia de este oficial es una copia manuscrita del siglo XIV de Chipre que acompañaba los Assises y fue titulado como Abrégé du libre des Assises de le Cour des bourgeois (Beugnot, 1841-1843, pp. 237, 238, 243-244). 
designaba al oficial latino, con aquel del mundo musulmán, el muhtasib. Sus funciones eran las mismas y la similitud en la denominación de uno y otro señala el esfuerzo por adaptar el término árabe al francés antiguo. Por lo tanto, se habría tratado de un funcionario que los francos heredaron de la administración y legislación árabe, razón que brindó un argumento sólido para pensar que la práctica médica que los cruzados desarrollaron en Tierra Santa era básicamente una adaptación del modelo musulmán (Conrad, 1999, p. XLIX). Sin embargo, este aspecto no resulta exclusivo del mundo de Outremer. La figura del muhtasib fue replicada, lógicamente, en muchos de los territorios que estuvieron bajo el dominio y la administración árabe. ${ }^{13} \mathrm{Al}$ igual que en los territorios cruzados se trató de una adaptación del término árabe. De esta forma, resulta problemático asumir una relación lógica entre la regulación médica de los Assises y el Hisba a partir de estos préstamos idiomáticos.

Del mismo modo, si se asume como válida dicha relación, es llamativo que los estándares de conocimiento teórico médico que se solicitaban en los Assises no eran necesariamente los más sofisticados, como sí los que se solicitaban en el texto musulmán. En efecto, la información que podemos extraer de un ejemplar de las hisbah, (Buckley, 1999) es que el conocimiento y los procedimientos médicos, tanto en la evaluación de un profesional como en las tareas que él debía desarrollar, eran comparativamente más elevados que los señalados en los Assises. Además, existían importantes diferencias entre el texto franco y el texto musulmán. En primer lugar, la forma en la que se enunciaba la norma, así como los castigos para los médicos que cometieran "mala praxis". En segundo lugar, la información y las descripciones en la hisbah sobre terapias, tipos de enfermedades, tipos de profesionales, autores y manuales que los médicos debían conocer se encontraban significativamente más desarrolladas que en los Assises (Buckley, 1999, pp. 116-117). No podemos, por lo tanto, deducir de los procedimientos indicados en los Assises un grado de elaboración teórica tan alto como el que se presenta en la hisbah.

\section{Enfermedades y respuestas sociales: el padecimiento de la lepra}

El abordaje de la historia de las enfermedades en el mundo antiguo y medieval proviene de diferentes campos. Desde la historia de la medicina, la historia social y la historia ambiental, existe una tradición de trabajos que se han ocupado de analizar las respuestas dadas hacia algún tipo de padecimiento en el mundo antiguo y medieval (McNeil, 2016 [1978]; Sallares, 2014; Headrick, 2012). De la misma forma, desde diferentes campos se hizo uso de un conjunto diverso de técnicas para entender qué enfermedades existieron y cómo se las trataba en los estados de Outremer (Anastasiou y Mitchell, 2015; Mitchell, 2015; Edgington, 1994).

A propósito de la circulación de saberes y prácticas médicas relativas al entendimiento de la enfermedad en el Reino Latino de Jerusalén, los primeros estudios de este campo ponderaron una visión que destacó la inferioridad de la medicina desplegada por los europeos en comparación con la que existía en Tierra Santa gracias a la tradición local siríaca o árabe (Ell, 1996; Ficarra, 1996). Del mismo modo, la revisión de estas lecturas permitió recalibrar esa imagen de rechazo e inferioridad del conocimiento europeo. Las coordenadas historiográficas en este punto son, en términos generales, las mismas que enunciamos a propósito de los médicos de las cruzadas. En cambio, un aspecto que requiere un análisis particular es el estudio del tipo de respuestas que socialmente existieron en torno al sujeto enfermo de lepra y como se lo trató en el Reino Latino de Jerusalén. 
Entendida hoy como una enfermedad infecciosa bacteriana, ${ }^{14}$ a lo largo de la historia, la palabra "lepra" fue utilizada genéricamente para describir una serie de diferentes infecciones que afectaban la piel de manera notoria (McNeil, 2016 [1978]; p. 174). Hoy llamada "enfermedad de Hansen", el tratamiento de la lepra posee un lugar central dentro del conjunto de los estudios históricos de la enfermedad (Demaitre, 2007; Rawcliffe, 2006; Touati, 1996, 1998; Brody, 1974).

En relación con la comprensión de la lepra en las sociedades medievales del mundo europeo occidental, durante mucho tiempo se destacó en la historiografía la idea de rechazo y estigmatización. Como indicó Bruno Tabuteau, heredamos de la Ilustración la imagen de una Edad Media sombría, identificada con esta enfermedad, muchas veces asociada a una de las tantas consecuencias nefastas de las cruzadas (Mitchell, 2002; Foucault, 1988, p. 6). Semejante imagen pervivió en Occidente por 200 años. En efecto, la renovación de este campo de estudios es reciente. A partir de la obra de François-Olivier Touati $(1998,1996)$ pudo surgir una relectura del leproso, las leproserías y la relación que la sociedad mantuvo con la lepra durante la Edad Media y una nueva interpretación basada en la lectura de las fuentes y su investigación crítica. Efectivamente, producto de su trabajo, se comenzó a reevaluar la idea de exclusión del leproso y la función segregativa de las leproserías en la Edad Media. La imagen de esta última institución como una prisión era reemplazada por la de una existencia conventual donde los leprosos constituían hermandades, económica y socialmente integradas al resto de la sociedad. ${ }^{15}$

En el mundo del islam, parecería haber existido una dinámica similar a la que la historiografía más reciente describió para el mundo occidental (Dols, 1983, p. 915). Sobre el mundo bizantino, se ha insistido acerca del carácter distintivo del tratamiento al leproso y las consideraciones positivas hacia la enfermedad (Miller y Nesbitt, 2014, pp. 40-41; 52-58) expresada en una actitud de inclusión y tolerancia hacia el leproso por parte de los primeros padres de la iglesia bizantina y la creación de un gran número de leprosarios desde el siglo IV, producto de la filantropía de muchos emperadores o miembros de la familia real y la iglesia griega (Constantelos, 1968; Miller y Nesbitt, 2014, pp. 72-73; Zias, 1986). ${ }^{16}$

Para el mundo de Outremer se ha destacado una sensibilidad particular sobre el padecimiento de la lepra, donde semejante condición era vista muchas veces de manera positiva (Barber, 1994; Kedar, 1983; Mitchell, 2000; Shahar, 1982). Para algunos autores, el rol de los Caballeros de la Orden de San Lázaro y su importancia en la defensa del reino de Jerusalén fue fundamental para esta apreciación del leproso (Barber, 1994; Hyancinthe, 2007). Otros sostuvieron que dicha valoración habría derivado de la famosa historia del rey Balduino IV. Según Piers Mitchell, la imagen del rey sabio que gobernó de manera prudente, dedicada y justa dio forma a una valoración positiva hacia el padecimiento de esta enfermedad (Mitchell, 2000). Mitchell, al igual que Shulamith Shahar (1982) casi 20 años antes que él, arribaba a esta conclusión a partir del análisis de una serie de pasajes sobre la vida y obra de Balduino de la crónica de Guillermo de Tiro. ${ }^{17}$

14 Los mecanismos patogénicos de la enfermedad no se conocen con exactitud. De hecho, la mayoría de las personas son inmunes a la lepra. Se estima que solo un $10 \%$ de la población puede sufrir la enfermedad (Demaitre, 2007, pp. vii-xii).

15 En este punto, la historiografía destacó el valor cuasi monacal de las leproserías (Tabuteau, 2007, p. 44; Hyacinthe, 2007, p. 214).

16 Touati reveló la existencia de 91 lugares de cuidado y asistencia en Tierra Santa entre el siglo IV y el año 1291, momento de expulsión de los cruzados de Outremer (Touati, 2007, p. 170).

17 Véase el famoso pasaje de la historia de Guillermo de Tiro sobre el padecimiento de la lepra del joven Balduino IV (Huygens et al., 1986, pp. 961-962). 
Para Rafaël Hyacinthe, en cambio, la importancia del "rey leproso" en la sociedad latina de Ultramar derivaba de la debilidad del Reino Latino hacia finales del siglo XII. ${ }^{18}$ Según este autor, los nobles eran conscientes de la fragilidad dinástica y la necesidad de sostenimiento de Balduino, único heredero de su padre el rey Almarico I, para mantener la unidad del Reino Latino de Jerusalén (Hyacinthe, 2007, p. 216). Hyacinthe retomaba -aunque despojado de gran parte de la teoría que lo sustentabalos argumentos esgrimidos por Mark Gregory Pegg en un artículo titulado Le corps et l'autorité: la lépre de Baaudouin IV. El autor analizaba la particularidad del rey leproso y cómo la metáfora del juicio del cuerpo del monarca, desarrollada en Europa, no se aplicaba a Balduino IV (Pegg, 1990).

Si el cuerpo constituía un microcosmos, relato de la realización de Dios en el interior del individuo, ¿cómo explicar entonces la imagen positiva que se sostenía de Balduino y su cuerpo enfermo de lepra? Para Pegg, la razón fundamental residía en que, a diferencia de lo que ocurría en el mundo occidental, en el Oriente latino el poder del monarca se encontraba fuertemente descentralizado. En efecto, desde la década del ' 50 del siglo XII no era la corte real donde residía el verdadero centro de mando sino en la hautecour. Por lo tanto, en la realidad del reino de Jerusalén el leproso no significaba, o no representaba, una amenaza a la integridad del cuerpo social. El verdadero problema residía en que el grupo de la nobleza, verdadera detentadora del poder, pudiera desunirse. Este factor explicaba que los leprosos de los estados cruzados pudieran ser aceptados en los altos círculos de la sociedad y que pudiera existir una orden de caballería de leprosos como los lazaristas. Del mismo modo, solo así era posible entender que el problema con Balduino IV no era su cuerpo enfermo, todo lo contrario, sino la desunión o la separación del grupo de la nobleza. Esa era la verdadera metáfora del Reino Latino de Jerusalén. Preservar al rey, por lo tanto, era preservar ya no su cuerpo sino el que integraba la nobleza (Pegg, 1990).

Para Hyacinthe lo importante del trabajo de Pegg era situar la dimensión política en la construcción positiva que las crónicas realizaron sobre el rey leproso. Dicha construcción, por lo tanto, no derivaría necesariamente en juicios de valor positivos hacia el leproso, sino de una necesidad política coyuntural específica (Barber, 1981). Del mismo modo, hacer extensiva la historia de Guillermo de Tiro sobre Balduino IV hacia el conjunto de la sociedad latina en Outremer parece un procedimiento poco fiable ya que el contenido de la misma posee un compromiso sentimental del autor hacia el joven príncipe.

De este modo, si uno atiende a otros elementos médico-institucionales puede pensar que una valoración del tipo particular que existió en el reino latino podría derivar de diversos factores culturales que nos llevarían por canales poco explorados. Quizás en el desarrollo de esta sensibilidad particular del leproso tuvo mucho peso la presencia de la iglesia bizantina en Tierra Santa. ${ }^{19}$ De la misma manera, el rol de los caballeros de San Lázaro y la atención monástica desplegada por la orden hacia los leprosos pudo también influir en este aspecto (Marcombe, 2003, p. 1).$^{20}$ Lo cierto es que este es un campo sobre el que todavía queda mucho por indagar.

18 Balduino IV fue coronado en el año 1174 y gobernó hasta el momento de su muerte en el año 1185 . Durante su reinado, además de disputas políticas internas entre los barones del reino, tuvo que atender al avance musulmán sobre sus fronteras

19 La historiografía describió cómo en el mundo bizantino prevaleció desde los primeros siglos de su existencia la preocupación por la incorporación del leproso dentro de la vida civil. Su cuidado, al mismo tiempo, fue una empresa que la iglesia griega desplegó en sus monasterios no solo en Constantinopla sino también en ciudades como Antioquía o Jerusalén (Ashkenazi, 1999).

20 Existen pocos trabajos que hayan atendido particularmente a la historia de la orden en Tierra Santa. Algunos de los más significativos: Barber (1994), Hyacinthe (2007), Marcombe (2003), Savona-Ventura (2008). 


\section{Conclusiones}

El mundo de las cruzadas y del Oriente latino constituye un escenario privilegiado para el estudio de la circulación de conocimientos y prácticas científicas ya que en los dos siglos de su existencia confluyeron de manera contundente instituciones y sujetos que portaban saberes e ideas de tradiciones diversas y distantes. El análisis de la evidencia documental que poseemos de la práctica médica desplegada en los estados cruzados permitió a los especialistas la construcción de toda una serie de imágenes históricas sobre quienes protagonizaron dicho despliegue. Muchas de ellas han sido revisadas recientemente y, como vimos, mucho queda aún por estudiar. Nuestro repaso historiográfico sobre los grandes temas en los que puede ser comprendido este campo de estudios derivó en una serie de conclusiones. A modo de balance, en primer lugar, observamos la centralidad otorgada a las terapias dispuestas (fundamentalmente a partir de las dietas prescriptas) por órdenes militares para indicar la procedencia de los conocimientos médicos, así como la importancia del mundo institucional bizantino en el mundo latino de Outremer. En segundo lugar, insistimos desde un conjunto de lecturas sobre la importancia de revertir la afirmación acerca de la inferioridad y el rechazo de los europeos por el conocimiento local, así como la supuesta inferioridad profesional. En tercer lugar, a propósito de la legislación médica contenida en los Assises observamos que, según más de un autor, el modelo de referencia para su redacción derivó del texto de la administración árabe, la hisba y la legislación que contenía en materia médica. En efecto, lo más lógico sería que este texto fuera un punto de referencia para los juristas francos en las ciudades que durante más de cuatro siglos, antes de la conquista cruzada, estuvieron bajo dominio musulmán. Sin embargo, las diferencias de contenido y su grado de elaboración teórica, así como la estructura de enunciación de la norma, son muy importantes para indicar una relación directa. Cuarto, y, por último, en el Reino Latino de Jerusalén prevaleció una actitud de inclusión del leproso y una valoración positiva hacia la enfermedad, aunque no haya una idea dominante sobre la causa de tal valoración. 


\section{Dibliografía}

» Albarrán Iruela, J. (2017). El sueño de al-Quds. Los musulmanes ante la conquista cruzada de Jerusalén (1099-1187). Madrid: La Ergástula.

"Amouroux, M. (1999). Colonization and Creation of Hospitals: the Eastern Extension of Western Hospitality in the Eleventh and Twelfth Centuries. Mediterranean Historical Review, 14, 31-43.

»Anastasiou, E. y Mitchell, P. D. (2015). Human Intestinal Parasites and Dysentery in Africa and the Middle East prior to 1500. En P. D. Mitchell (Ed.), Sanitation, Latrines and Intestinal Parasites in Past Populations (121-147). Farnham: Ashgate.

"Ashkenazi, Y. (1999). Curing and Nursing in the Church of Jerusalem during the Byzantine Period. En A. Zohar, E. Lev y J. Schwartz (Eds.), Medicine in Jerusalem throughout the Ages (33-49). Tel Aviv: Eretz.

» Barber, M. (1981). Lepers, Jews and Moslems: the Plot to overthrow Christendom in 1321. History, 66, 1-17.

» Barber, M. (1994). The Order of Saint Lazarus and the Crusades. The Catholic Historical Review, LXXX, 439-456.

" Barber, M. (2000). The Charitable and Medical Activities of the Hospitallers and Templars. En G. Evans (Ed.), A History of Pastoral Care (148-168). Londres: Bloomsbury Publishing.

» Beugnot, A. (Ed.). (1841-1843). Recueil des historiens des Croisades: Lois, 2. Abrégé du livre des Assises de le Cour des bourgeois. Paris: Académie Royale des Inscriptions et BelleLettres.

» Bouras-Vallianatos, P. y Zipser, B. (Eds.). (2019). Brill's Companion to the reception of Galen. Leiden- Boston: Brill.

" Brody, S. N. (1974). The Disease of the Soul: Leprosy in Medieval Literature. Nueva York: Cornell University Press.

» Buckley, R. P. (Trad.). (1999). The Book of the Islamic Market Inspector: Nihayat al-rutba fi talab al-hisba (The Utmost Authority in the Pursuit of Hisba) by 'Abd al-Rahman b. Nasr al-Shayzari. Journal of Semitic Studies Supplement, 9, 1-217.

»Burnett, C. (2000). Antioch as a Link between Arabic and Latin Culture in the Twelfth and Thirteenth Centuries. En B. Van Den Abeele, A. Tihon e I. Draelants (Eds.), Occident et Proche-Orient: contacts scientifiques au temps des Croisades. Actes du colloque de Louvain-la-Neuve, 24 et 25 mars 1997 (1-69). Turnhout: Brepols.

"Cahen, C. (1934). Indigènes et croisés. Quelques mots à propos d'un médecin d'Amaury et de Saladin. Syria, 15(4), 351-360.

» Ciggaar, K. (2001). Western Travelers to Constantinople. The West and Byzantium, 962-1204: Cultural and Political Relations. Leiden: Brill.

» Ciggaar, K., Teule, H. y Davis, A. (Eds.). (1996-2003). East and West in the Crusader States: Context. 3 vols. Lovaina: Peeters Publishers.

»Conrad, L. (1999). Usama ibn Munqidh and Other Witnesses to Frankish and Islamic Medicine in the Era of the Crusades. En Z. Amar, E. Lev y J. Schwartz (Eds.), Medicine in Jerusalem throughout the Ages (xxvii-lii). Tel Aviv: Eretz.

"Constantelos, D. J. (1968). Byzantine philanthropy and social welfare. Nueva Brunswick y Nueva Jersey: Rutgers University Press. 
» D’Alverny, M.-T. (1982). Translations and Translators. En R. L. Benson y G. Constable (Eds.), Renaissance and Renewal in the Twelfth Century (421-462). Cambridge: Cambridge University Press.

» Demaitre, L. (2007). Leprosy in Premodern Medicine: A Malady of the Whole Body. Baltimore: Johns Hopkins University Press.

»Dolev, E. (1996). Medicine in the Crusaders Kingdom of Jerusalem. En M. Waserman y S. Kottek (Eds.), Health and Disease in the Holy Land: Studies in the History and Sociology of Medicine from Ancient Times to the Present (157-172). Nueva York y Lampeter: The Edwin Mellen Press.

» Dols, M. (1983). The Leper in Medieval Islamic Society. Speculum, 58(4), 891-916.

» Edgington, S. (1994). Medical Knowledge in the Crusading Armies: the Evidence of Albert of Aachen and Others. En M. Barber (Ed.), The Military Orders: Fighting for the Faith and Caring for the Sick (320-326). Londres: Ashgate.

» Edgington, S. (1998). Medical Care in the Hospital of St John in Jerusalem. En H. Nicholson (Ed.), The Military Orders: Fighting for the Faith and Caring for the Sick (Vol. 2, 27-33). Londres: Ashgate.

» Edgington, S. (1999). The Hospital of St John in Jerusalem. En Z. Amar, E. Lev y J. Schwartz (Eds.), Medicine in Jerusalem throughout the ages (ix-xxv). Tel Aviv: Eretz.

» Edgington, S. (2005a). Administrative Regulations for the Hospital of St John in Jerusalem dating from the 1180s. Crusades, 4, 21-37.

"Edgington, S. (2005b). Medicine and surgery in the Livre des Assises de la Cour des Bourgeois de Jerusalem. Al-Masāq, Islam and the Medieval Mediterranean, 17(1), 87-97.

»Edgington, S. (2005c). Medieval Antioch as an Intellectual Centre, and its Influence on Western European Medicine. En Sari, N. et al. (Eds.), Proceedings of the 38th International Congress on the History of Medicine, 1-6 September 2002 (481-87). Ankara: Türk Tarih Kurumu.

» Edgington, S. (2011). Oriental and occidental medicine in the crusader states. En Kostick, C. (Ed.), The Crusades and the Near East: Cultural Histories (189-215). Londres: Routledge.

»Elisseef, N. (1986). Les échanges culturels entre le monde musulman et les croisés à l'époque de Nurad-Din b. Zanki (m. 1174). En V. P. Goss y C. Verzar Bornstein (Eds.), The Meeting of two worlds: cultural exchange between East and West during the period of the Crusades (39-52). Michigan: Medieval Institute Publications, Western Michigan University.

» Ell, S. (1996). Pilgrims, Crusades and Plagues. En M. Waserman y S. Kottek (Eds.), Health and disease in the Holy Land: studies in the history and sociology of medicine from ancient times to the present (173-187). Nueva York y Lampeter: The Edwin Mellen Press.

» Ficarra, B. (1996). Disease to death during the Crusades. En M. Waserman y S. Kottek (Eds.), Health and disease in the Holy Land: studies in the history and sociology of medicine from ancient times to the present (135-155). Nueva York y Lampeter: The Edwin Mellen Press.

» Foucault, M. (1988). Madness and civilization. Nueva York: Vintage Books.

» Gabrieli, F. (1969). Arab Historians of the Crusades. Londres: Routledge and Kegan Paul.

» Handerson, H. (1918). Gilbertus Anglicanus: Medicine of the Thirteen Century. Cleveland: Cleveland Medical Library Association.

» Haskins, C. (1925). Arabic Sciences in Western Europe. Isis, 7(3), 478-85.

» Headrick, D. (2012). The medieval World, 500 to 1500 CEE. En J. R. McNeill y E. Stewart 
Mauldin (Eds.), A companion to Global Environmental History (39-56). West Sussex: Wiley Blackwell.

» Hillebrand, C. (1999). The Crusades. Islamic Perspectives. Edimburgo: Edinburgh University Press.

» Hitti, P. (Ed.). (1930). Usama Ibn Munqidh. Kitāb al-l'tibār. Princeton: Princeton University Press.

» Hitti, P. (Trad.). (1929). An Arab-Syrian Gentleman and Warrior in The Period of The Crusades: Memoirs of Usama Ibn-Munqidh (Kitab al i'tibar). Nueva York: Columbia University Press.

» Hume, E. (1940). Medical Work of the Knights Hospitallers of Saint John of Jerusalem. Baltimore: Johns Hopkins Press.

» Huygens, R. et al. (Eds.). (1986). Guillaume du Tyr. Chronicon. Turnhout: Brepols. (CCCM).

» Hyacinthe, R. (2007). De Domo Sancti Lazari milites leprosi: Knighthood and Leprosy in the Holy Land. En B. Bowers (Ed.), The Medieval Hospital and Medical Practice (209-224). Londres: Ashgate.

» Kedar, B. (1983). Gerard of Nazareth a Neglected Twelfth-Century Writer in the Latin East: A Contribution to the Intellectual and Monastic History of the Crusader States. Dumbarton Oaks Papers, 37, 55-77.

» Kedar, B. (1998). A Twelfth-Century Description of the Jerusalem Hospital. En H. Nicholson (Ed.), The Military Orders: Fighting for the Faith and Caring for the Sick (Vol. 2, 3-26). Londres: Ashgate.

» Luttrell, A. (1994). The Hospitallers Medical Tradition: 1291-1530. En M. Barber (Ed.), The Military Orders: Fighting for the Faith and Caring for the Sick (64-81). Londres: Ashgate.

" Marcombe, D. (2003). Leper Knights. The Order of St Lazarus of Jerusalem in England, C.1150-1544. Woodbridge: The Boydell Press.

» McNeil, W. (2016 [1978]). Plagas y pueblos. Madrid: Siglo XXI.

» Micheau, F. (2000). Les médecins orientaux au service des princes latins. En Van Den Abeele, B., Tihon, A. y Draelants, I. (Eds.), Occident et Proche-Orient: contacts scientifiques au temps des Croisades. Actes du colloque de Louvain-la-Neuve, 24 et 25 mars 1997 (95-115). Turnhout: Brepols.

» Miller, T. (1978). The Knights of St John and the Hospitals of the Latin West. Speculum, 53(4), 709-733.

" Miller, T. y Nesbitt, J. (2014). Walking corpses. Leprosy in Byzantium and the Medieval West. Ithaca y Londres: Cornell University Press.

» Mitchell, P. D. (2000). Appendix: An Evaluation of the Leprosy of King Baldwin IV of Jerusalem in the Context of Medieval World. En B. Hamilton (Ed.), The Leper King and his Heirs. Baldwin IV and the Crusader Kingdom of Jerusalem (245-258). Nueva York: Cambridge University Press.

» Mitchell, P. D. (2002). The Myth of the Spread of Leprosy with the Crusades. En C. A. Roberts, K. Manchester y M. E. Lewis (Eds.), The Past and Present of Leprosy (171-178). Oxford: Archaeopress.

» Mitchell, P. D. (2004). Medicine in the Crusades: Warfare, Wounds and the Medieval Surgeon. Cambridge: Cambridge University Press.

» Mitchell, P. D. (2007). The Infirmaries of the Order of the Temple in the Medieval Kingdom of Jerusalem. En B. Bowers (Ed.), The Medieval Hospital and Medical Practice (225234). Londres: Ashgate. 
» Mitchell, P. D. (2015). Intestinal Parasites in the Crusades: Evidence for Disease, Diet, and Migration. En A. Boas (Ed.), The Crusader World (593-606). Londres y Nueva York: Routledge, 2015.

» Mitchell, P. D. (2016). Anatomy and Surgery in Europe and the Middle East During the Middle Ages. En H. Perdicoyianni-Paleologou (Ed.), Anatomy and Surgery from Antiquity to the Renaissance (309-324). Amsterdam: Adolf Hakkert.

» Nader, M. (2006). Burgesses and Burgess Law in the Latin Kingdoms of Jerusalem and Cyprus (1099-1325). Hampshire: Ashgate.

»Pegg, M. G. (1990). Le corps et l'autorité: la lèpre de Baudouin IV. Annales. Économies, Sociétés, Civilisations. 45(2), 265-287.

» Pormann, P. y Savage-Smith, E. (2007). Medieval Islamic Medicine. Edimburgo: Edinburgh University Press.

»Prawer, J. (1980). Crusader Institutions. Oxford: Oxford University Press.

» Rawcliffe, C. (2006). Leprosy in Medieval England. Woodbridge: Boydell Press.

»Sallares, R. (2014). Disease. En P. Horden y S. Kinoshita (Eds.), A companion to Mediterranean History (250-262). West Sussex: Wiley Blackwell.

»Savage-Smith, E. (2006). New Evidence for the Frankish Study of Arabic Medical Texts in the Crusader Period. Crusades, 5, 99-112.

»Savona-Ventura, C. (2008). The Order of Saint Lazarus in the Kingdom of Jerusalem. Journal of the Monastic Military Orders, 1, 55-64.

»Shahar, S. (1982). Des lépreux pas comme les autres. L'Ordre de Saint-Lazare dans le royaume latin de Jérusalem. Revue historique, 267, 19-41.

"Siraisi, N. (1990). Medieval and Early Renaissance Medicine. Chicago: University of Chicago Press.

»Sterns, I. (1983). Care of the Sick Brothers by the Crusader Orders in the Holy Land. Bulletin of the History of Medicine, 57, 43-69.

»Tabuteau, B. (2007). Historical Research Developments on Leprosy in France and Western Europe. En B. Bowers (Ed.), The Medieval Hospital and Medical Practice (41-56). Londres y Nueva York: Routledge.

» Temkin, O. (1973). Galenism. Rise and Decline of a Medical Philosophy. Ithaca y Londres: Cornell University Press.

» Torres Fontes, J. (1983). Las ordenaciones al Almotacen murciano en la primera mitad del siglo XIV. Miscelánea medieval murciana, 10, 71-131.

» Touati, F.-O. (1996). Archives de la lèpre. Atlas des léproseries entre Loire et Marne au Moyen Age. Paris: Editions du Comité des travaux historiques et scientifiques.

» Touati, F.-O. (1998). Maladie et société au Moyen Âge. La lèpre, les lépreux et les léproseries dans la province ecclésiastique de Sens jusqu'au milieu du XIVe siècle. Turnhout: Brepols.

» Touati, F.-O. (2007). La Terre Sainte: un laboratoire hospitalier au Moyen Âge? En N. Bulst y K. H. Heinz (Eds.), Sozialgeschichte Mittelalterlicher Hospitäler (169-211). Ostfildern: Jan Thorbecke Verlag.

»Van Den Abeele, B., Tihon, A. y Draelants, I. (2000). Introduction. En B. Van Den Abeele, A. Tihon e I. Draelants (Eds.), Occident et Proche-Orient: contacts scientifiques au temps des Croisades. Actes du colloque de Louvain-la-Neuve, 24 et 25 mars 1997 (i-v). Turnhout: Brepols.

»Wagner, T. G. (2009). Die Seuchen der Kreuzzüge: Krankheit und Krankenpflege auf den 
bewaffneten Pilgerfahrten ins Heilige Land. Würzburg: Königshausen \& Neumann.

»Walsh, J. (1919). The Medical History of Two Crusades. En Contributions to Medical and Biological Research, Dedicated to Sir William Osler (Vol. 2, 796-805). Nueva York: Paul B. Hoeber.

"Wickersheimer, E. (1936). Dictionnaire biographique des médecins en France au Moyen Âge. Paris: E. Droz.

»Wickersheimer, E. (1951). Organisation et législation sanitaires au royaume franc de Jerusalem (1099-1296). Archives internationales d'histoire des sciences, 16, 689-705.

»Woodings, A. F. (1971). The Medical Resources and Practice of the Crusader States in Syria and Palestine, 1096-1193. Medical history, 15, 268-277.

»Zias, J. (1986). Was Byzantine Herodium a Leprosarium? The Biblical Archaeologist, 49(3), 182-186. 